\title{
Atherosclerotic lesion formation in rabbits fed on egg yolk-supplemented diet: an inexpensive experimental model
}

\author{
Formação de lesão aterosclerótica em coelhos alimentados com dieta \\ suplementada com gema de ovo: modelo experimental de baixo custo
}

The paper "Atherosclerotic lesion formation in rabbits fed on egg yolk-supplemented diet: an inexpensive experimental model" published in this journal in December 2006 was directed at finding an animal model that presented similar characteristics to human atherosclerosis. Several efforts have been made to find this ideal model, and all studied species have both strengths and limitations. In the aforementioned paper, atherosclerosis was induced by feeding rabbits with egg yolk for 90 days. Although its cost is lower than other methods described in the paper, the atherosclerotic lesions obtained with egg supplementation were quite modest. It was not clear if there were significant differences in lipid profile between the control and treated groups. However, even if significant increases in plasma lipids were observed in the animals submitted to the egg diet, histological findings were not robust.

Mild atherosclerotic lesions might have been due to the effects of egg yolk in lipid metabolism. There are a number of studies demonstrating that egg yolk is able to promote an increase in either plasma HDL-cholesterol (HDL-c) and in LDL-cholesterol (LDL-c). ${ }^{1,2}$ Even individuals who are hyper-responsive to dietary cholesterol challenge do not present worsening in LDL-c/ HDL-c ratio, which is a better predictor of cardiovascular disease than each of them isolated. ${ }^{3,4}$ In addition, considering LDL particle sizes, egg intake favors the

Artigo submetido em 11.05.07, aceito em 29.05.07.

J Vasc Bras 2007;6(2):197-200.

Copyright $\odot 2007$ by Sociedade Brasileira de Angiologia e de Cirurgia Vascular change from small-dense LDL pattern (pattern B) to large LDL pattern (pattern A), which are much less susceptible to oxidation and to deposition in vascular wall. ${ }^{5}$ Furthermore, adjustment by body weight should also be considered to determine whether obesity was a determinant factor promoting lipid alterations and, consequently, histological findings.

A cheap and efficient option to induce atherosclerosis would be the use of another animal model, for example Hartley guinea pigs. ${ }^{6}$ Considering the two most common breeds of both species, the price of each guinea pig is much cheaper than New Zealand white rabbits (from US\$ 46 to 78 for each guinea pig and from US $\$ 80$ to 169 for each rabbit) in the USA. ${ }^{7}$ In addition, the price of feeding guinea pigs is at least comparable to the price of feeding rabbits. ${ }^{8}$ For example, in our lab the final cost of the semi-purified diet used to feed one guinea pig with high cholesterol and high carbohydrate diet for 12 weeks was about US\$ 73.5. In the USA, based on a non-enriched chow, the cost of feeding each rabbit would vary from about US\$ 79 to 119 for the same period of time. ${ }^{8}$ Hence, in the USA, the combined cost of buying and feeding rabbits is actually higher when compared to guinea pigs. ${ }^{9,10}$ It is possible that the costs of buying and feeding guinea pigs are cheaper than rabbits also in Brazil. However, possible variations in price among countries might be expected and should be considered.

In addition to the possible advantages in cost, guinea pigs might be a better surrogate to human atherosclerosis than rabbits. Physiologically, guinea pigs present the 
closest lipid metabolism to humans. ${ }^{6}$ Most of their plasma cholesterol is carried in LDL, as opposed to rodents and rabbits, which carry most of cholesterol in HDL and very low-density lipoprotein (VLDL) particles, respectivelly. ${ }^{6,9}$ Guinea pigs present similar activities in the major regulators of cholesterol and lipoprotein metabolism, including lecithin:cholesterol acyltransferase (LCAT), cholesteryl ester transfer protein (CETP), acyl-coenzyme A:cholesterol acyl transferase (ACAT), cholesterol 7alpha-hydroxylase (CYP7) and 3-hydroxy-3-methyl-glutaryl-CoA reductase (HMG-CoA reductase) compared to humans. ${ }^{6}$ They respond very well to cholesterol, carbohydrate, fatty acids, including trans-fatty acids and polyphenols challenge. ${ }^{6}$ Because of the aforementioned similarities to humans, guinea pigs have been chosen as the prototype to study drugs that interfere with atherosclerosis and lipid metabolism, for example, HMG-CoA reductase inhibitors, cholestyramine, apical sodium-dependent bile acid transporter (ASBT) inhibitor, microsomal triglyceride transfer protein (MTP) inhibitor and rapamycin. ${ }^{6,10}$ Hence, the use of guinea pigs should be considered when the aim is to have diet-induced atherosclerosis.

On the other hand, the great disadvantage of guinea pigs is exactly the biggest strength of rabbits. Whilst the literature considering guinea pigs as surrogate to surgical models is quite little, there is a vast number of experiments using rabbits. These animals became a classic model to study restenosis especially after the development of balloon and wire injury models. ${ }^{11,12}$ Therefore, rabbits might be a superior model when the aim is to study intimal hyperplasia or surgical techniques.

Others simple interventions, as described by the authors, could induce atherosclerosis more severely in the rabbit model. An increasing carbohydrate and saturated fat content on chow would be an inexpensive alternative. The corn oil used to enrich the chow could be replaced by coconut oil. The idea would be to replace the oil rich in polyunsaturated fatty acids, which does not accelerate atherosclerotic lesions, by an oil rich in saturated fatty acids, which is extremely atherogenic, without increasing the costs. ${ }^{13}$

In conclusion, although rabbits are a viable model to study atherosclerosis, other diet compositions or animal models could be used to strengthen the current results.

\section{José Oyama Moura Leite}

MD. Vascular surgeon and graduate student, Department of Nutritional Sciences, University of Connecticut, Storrs, CT, USA.

E-mail: joseoyama@hotmail.com

\section{Maria-Luz Fernadez}

PhD. Professor, Department of Nutritional Sciences, University of Connecticut, Storrs, CT, USA.

\section{References}

1. Greene CM, Zern TL, Wood RJ, et al. Maintenance of the LDL cholesterol: HDL cholesterol ratio in an elderly population given a dietary cholesterol challenge. J Nutr. 2005; 135:2793-8.

2. Ballesteros MN, Cabrera RM, Saucedo Mdel S, Fernandez ML. Dietary cholesterol does not increase biomarkers for chronic disease in a pediatric population from Northern Mexico. Am J Clin Nutr. 2004;80:855-61.

3. Herron KL, Vega-Lopez S, Conde K, Ramjiganesh T, Shachter NS, Fernandez ML. Men classified as hypo- or hyperresponders to dietary cholesterol feeding exhibit differences in lipoprotein metabolism. $\mathbf{J}$ Nutr. 2003; 133:1036-42.

4. Herron KL, Vega-Lopez S, Conde K, et al. Pre-menopausal women, classified as hypo- or hyperresponders, do not alter their LDL/HDL ratio following a high dietary cholesterol challenge. J Am Coll Nutr. 2002;21:250-8.

5. Herron KL, Lofgren IE, Sharman M, Volek JS, Fernandez ML. High intake of cholesterol results in less atherogenic low-density lipoprotein particles in men and women independent of response classification. Metabolism. 2004;53:823-30.

6. Fernandez ML, Volek JS. Guinea pigs: A suitable animal model to study lipoprotein metabolism, atherosclerosis and inflammation. Nutr Metab (Lond). 2006;3:17.

7. Charles River Laboratories, Inc [site on the Internet]. Wilmington, MA; c1995-2006. [updated 2006 Apr 28; cited 2007 May 09]. http://www.criver.com/index.html.

8. Dyets, Inc [site on the Internet]. Bethlehem, PA; c1999-2004. [updated 2006 Dec 17; cited 2007 May 09] http://www.dyets.com/pricing.htm.

9. Overturf ML, Loose-Mitchell DS. In vivo model systems: the choice of the experimental animal model for analysis of lipoproteins and atherosclerosis. Curr Opin Lipidol. 1992;3:179-85 
10. West KL, Fernandez ML. Guinea pigs as models to study the hypocholesterolemic effects of drugs. Cardiovasc Drug Rev. 2004;22:55-70.

11. Baumgartner HR. The role of blood flow in platelet adhesion, fibrin deposition, and formation of mural thrombi. Microvasc Res. 1973;5:167-79.

12. Walker LN, Ramsay MM, Bowyer DE. Endothelial healing following defined injury to rabbit aorta. Depth of injury and mode of repair. Atherosclerosis. 1983;47:123-30.

13. Narayanaswamy M, Wright KC, Kandarpa K. Animal models for atherosclerosis, restenosis, and endovascular graft research. J Vasc Interv Radiol. 2000;11:5-17.

\section{Resposta dos autores}

\section{Prezados Dr. Moura Leite e Dr. Fernandez,}

Agradecemos os comentários embasados e pertinentes que fizeram sobre o artigo "Atherosclerotic lesion formation in rabbits fed on egg yolk-supplemented diet: an inexpensive experimental model", publicado na edição de dezembro de 2006 do Jornal Vascular Brasileiro. Tal pesquisa foi realizada no intuito de avaliar a produção de aterosclerose experimental, através de fonte alimentar de colesterol de fácil acesso e baixo custo.

Estudamos a gema de ovo como fonte de colesterol alimentar, pois é um modelo clássico de indução de hipercolesterolemia. Além disso, a hipercolesterolemia alimentar é a via mais prática e difundida na indução de aterosclerose experimental. Ao contrário do afirmado na carta, a Figura 1 do artigo mostra claramente um aumento significante ao longo do tempo dos níveis sanguíneos de colesterol (LDL-colesterol e HDLcolesterol), exceto triglicérides, nos animais tratados em relação aos controles. Na discussão do artigo, são apontadas estratégias para se provocar hipertrigliceridemia nestes animais.

A espécie escolhida foi o coelho pois este é, indubitavelmente, o animal mais utilizado nos modelos de aterosclerose experimental ${ }^{1}$, e diversos estudos de impacto utilizaram este animal ${ }^{2,3}$. Sua utilização neste caso foi essencial, pois forneceu-nos elementos para comparação de resultados de outros autores. Além disso, o coelho é facilmente disponível nos biotérios e é usado em boa parte das pesquisas nesta área realizadas em nosso país e no mundo. A sugestão do uso de cobaias com certeza deve trazer contribuição adicional a este tipo de pesquisa. As vantagens apontadas na carta fazem crer que a cobaia passe a ocupar um lugar de crescente destaque neste tipo de pesquisa.

$\mathrm{Na}$ discussão do artigo, enfatizamos que as lesões ateroscleróticas observadas em nosso modelo foram similares às obtidas com modelos em que a quantidade de colesterol fornecida, por meio do colesterol comercial, era de $0,15 \%$. Modelos no mesmo animal com ingesta de $1,0 \%$ de colesterol na dieta mostraram lesões mais proeminentes. Assim, acreditamos que com fornecimento de maior proporção diária de gema de ovo, conseguiríamos lesões mais importantes sem impacto significante dos custos, em comparação com o colesterol purificado comercial, este sim, bastante oneroso: note-se que o custo da dieta com gema de ovo foi 81 vezes menor do que seria com colesterol comercial (Tabela 1 do artigo). Outras estratégias para incrementar as lesões foram igualmente apontadas na discussão do nosso trabalho. A sugestão dos missivistas de substituir o óleo de milho por óleo de coco é alternativa bastante atrativa e econômica.

As pesquisas com dietas e modelos de aterosclerose, como apontado na discussão do artigo, tiveram impulso desde o começo do século passado. Até hoje, há uma contínua busca por fórmulas e modelos que mimetizem as condições encontradas em seres humanos, com aplicabilidade e viabilidade. As pesquisas e os trabalhos desenvolvidos fornecem material fértil para discussão, sendo a polêmica e a contestação dos projetos essenciais para o enriquecimento do conhecimento. Agrademos aos autores da carta o interesse pelo tema do artigo e pela oportunidade desta troca de idéias.

\section{Rodrigo Gibin Jaldin}

Acadêmico de Medicina, Faculdade de Medicina de Botucatu (FMB), Universidade Estadual Paulista (UNESP), Botucatu, SP. 


\section{Winston Bonetti Yoshida}

Professor adjunto livre-docente, Disciplina de Cirurgia Vascular, Departamento de Cirurgia e Ortopedia, FMB, UNESP, Botucatu, SP.

\section{Referências}

1. Ramos Morales LE. La aterosclerosis: algunas consideraciones acerca de su estudio experimental; revisión bibliográfica. Rev Cuba Med. 1987;26:965-70.
2. Nicholls SJ, Cutri B, Worthley SG, et al. Impact of short-term administration of high-density lipoproteins and atorvastatin on atherosclerosis in rabbits. Arterioscler Thromb Vasc Biol. 2005;25:2416-21.

3. Bocan TM, Mueller SB, Mazur MJ, Uhlendorf PD, Brown EQ, Kieft KA. The relationship between the degree of dietary-induced hypercholesterolemia in the rabbit and atherosclerotic lesion formation. Atherosclerosis. 1993;102:9-22.

\section{Registro de Ensaios Clínicos}

O Jornal Vascular Brasileiro apóia as políticas para registro de ensaios clínicos da Organização Mundial da Saúde (OMS) e do International Committee of Medical Journal Editors (ICMJE; www.icmje.org), reconhecendo a importância dessas iniciativas para o registro e a divulgação internacional de informação sobre estudos clínicos, em acesso aberto. De acordo com essa recomendação, artigos de pesquisas clínicas devem ser registrados em um dos Registros de Ensaios Clínicos validados pelos critérios estabelecidos pela OMS e ICMJE, cujos endereços estão disponíveis no site do ICMJE (por exemplo, www.actr.org.au, www.clinicaltrials.gov, www.ISRCTN.org, www.umin.ac.jp/ctr/index/htm e www.trialregister.nl). O número de identificação deverá ser registrado ao final do resumo. A partir de 2008, somente serão aceitos para publicação artigos que tenham recebido um número de identificação nesses registros. 